\title{
Investigation of Laser Ablation on Acrylonitrile Butadiene Styrene Plastic Used for 3D Printing
}

\author{
Dongkyoung Lee*,† \\ *Department of Mechanical and Automotive Engineering, Kongju National University, \\ Cheonan, 31080, Korea
}

†Corresponding author : 1dkkinka@kongju.ac.kr

(Received June 16, 2017 ; Revised August 1, 2017 ; Accepted November 21, 2017)

\begin{abstract}
Microrobotic devices have been widely investigated as promising alternatives to convent biomedical technologies. Microrobotic devices having spatially complex structures such as the helical structures has generally been manufactured by using three-dimensional (3D) printing technologies. However, due to the limitations of the extrusion and lamination processes, microrobotic devices can be constructed with low degree of precision and can have poor surface roughness under the 3D printing technologies. This may cause undesirable damages to the human body tissues. Furthermore, 3D printing requires long processing time due to postprocessing to remove unwanted body parts. To improve surface quality and shorten processing time, this research uses laser machining. Before directly applying laser machining to fabricate microrobotic devices, understanding laser ablation characteristics on Acrylonitrile Butadiene Styrene (ABS), which is a common material used for 3D printing, is necessary. Laser ablation characteristics on this material are investigated with a variation of laser parameters such as a scanning speed and the number of scanning passes. An ablation depth prediction model is suggested. Laser ablation on ABS shows a high aspect ratio ( 327.2). Furthermore, color changes around the ablation zone can be utilized for the indication to estimate the absorbed laser energy indirectly.
\end{abstract}

Key Words : 3D printing, Laser machining, Thermoplastic polymer, Ablation characteristics, ABS, Micro-robotic devices

\section{Introduction}

Microrobotic devices have been widely studied as promising alternatives to conventional biomedical technologies ${ }^{1-3)}$. By utilizing magnetic materials, microrobotic devices can be wirelessly manipulated by external magnetic fields without the use of additional power transfer units or batteries. Thus, the overall structures of the microrobotic devices can be effectively minimized to be applied for the human body. Among various methods such as a magnetic force-based pulling method, cilia-like beating method, and helical (screwlike) propulsion method ${ }^{4-6)}$, the helical propulsion method is especially effective $e^{5)}$.

Conventional helical microrobotic devices have generally been manufactured by using three-dimensional (3D) printing technologies ${ }^{7}$. These technologies are useful when manufacturing spatially complex structures such as the helical structures. However, due to the limitations of the extrusion and lamination processes, microrobotic devices can be constructed with low degree of precision and can have poor surface roughness under the 3D printing technologies which may cause undesirable damages to the human body tissues. Furthermore, 3D printing requires long processing time due to postprocessing to remove unwanted body parts. To improve surface quality and shorten processing time, laser machining technology to fabricate helical structure could be a promising option. Laser-aided manufacturing processes including laser machining, laser cutting, and laser welding have been used actively due to their advantages such as non-contact process, fast processing speed, low Heat Affected Zone (HAZ), and high energy density ${ }^{8-18)}$. 
Laser machining is one of the active research field. Neuenschwander et. al. ${ }^{19)}$ investigated laser surface structuring with $100 \mathrm{~W}$ of average power and sub-picosecond pulses on a steel substrate. By using very high speeds of several $100 \mathrm{~m} / \mathrm{s}$ and repetition rates of several tenths of $\mathrm{MHz}$, an 'interlaced' mode can provide a maximum flexibility. Incubation effects depending on the number of pulses applied on two steel alloys during micromachining were studied ${ }^{20)}$. The phenomenological model was introduced and validated for prediction of complex ablation geometries in $\mathrm{NiCr}$ films using 10 ps laser puls$\mathrm{es}^{21)}$. Daniel et. al. ${ }^{22)}$ established an efficient method for a global parameter determination by varying power, repetition rate, scan speed, and track-distance of the laser process. Investigations were carried out by use of picosecond pulsed laser radiation on a Polycrystalline Cubic Boron Nitride (PCBN) grade with a high cubic boron nitride content and a titanium-based binder.

There are few research concerning ABS material using laser. Those few research are especially focused on the welding technology. Weldability of acrylonitrile/butadiene/ styrene (ABS) plates using laser beam is determined by combining both experimental and theoretical aspects ${ }^{23)}$. Acherjee et al. ${ }^{24)}$ studied diode laser transmission welding of dissimilar thermoplastics between Polymethyl methacrylate (PMMA) and ABS. In addition, to find the optmum solutions, a numerial multi-objective simultaneous optimization technique is incorporated and graphical optimization technique is implemented.

Applying laser machining to fabricate ABS plastic for micro-robotic devices have not been studied. Before directly apply the laser machining to the micro-robotic devices, this research focuses on ablation interaction characteristics between a laser and ABS plastic, used for $3 \mathrm{D}$ printing. The ABS is widely used for products such as musical instruments, golf club heads, automotive trim components, and automotive bumper bars due to its light weight and ability to be injection molded and extruded.

Laser ablation characteristics on the ABS are investigated with a variation of laser parameters such as a scanning speed and the number of scanning passes. First, this paper describes material preparation and experimental set-up. Second, results are analyzed and discussed. Moreover, a ablation depth prediction model is proposed. Finally, the conclusion is presented.

\section{Experiments}

A workpiece is prepared by Digital Light Processing (DLP) 3D printing. Acrylonitrile butadiene styrene (ABS) is used since this is common and widely used plastic for $3 \mathrm{D}$ printing. The $\mathrm{ABS}$ is a polymer compound and more specifically copolymer. This copolymer is built by chemical composition from acrylonitrile, butadiene, and styrene monomer units. The glass transition temperature of ABS is approximately $105{ }^{\circ} \mathrm{C}$. Due to the amorphous, there is no true melting point. Material properties of ABS are tabulated in Table 1. The ABS combines the strength and rigidity with the toughness, since acrylonitrile and styrene polymers provides the strength and rigidity as well as polybutadiene rubber provides the toughness. Furthermore, the ABS is considered superior for its hardness, gloss, toughness, and electrical insulation properties. While there are many available colors for the ABS, the ABS used for this study has yellow color. The thickness of workpiece is $10 \mathrm{~mm}$.

Experimental set-up is shown in Fig. 1. Diode pumped Q-switched solid state laser (ESI $5335^{\mathrm{TM}}$ ) is used. Wavelength $(\lambda)$ is $355 \mathrm{~nm}$ and spot size $(d)$ at focus is $15 \mu \mathrm{m}$. Repetition rate $(f)$ is $40 \mathrm{kHz}$. A pulse energy $(E)$ is $150 \mu \mathrm{J}$. A pulse duration $(\Delta t)$ is $120 \mathrm{~ns}$. The maximum average laser power is $6 \mathrm{~W}\left(\mathrm{P}_{\text {avg }}\right)$. Scanning speed $(v)$ varies in the range of 20 to $300 \mathrm{~mm} / \mathrm{s}$. For experiments, all laser parameters are set to constant except a scanning speed and the number of scan passes. Distance between

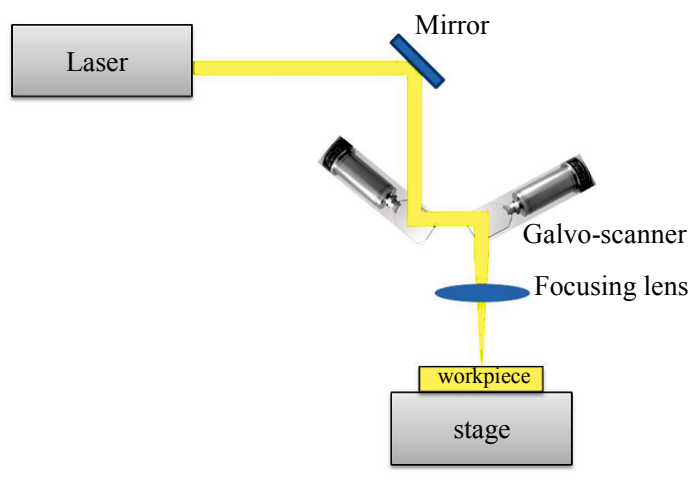

Fig. 1 Experimental set-up

Table 1 Material properties of ABS

\begin{tabular}{|c|c|c|}
\hline Classification & Value & Units \\
\hline Density & $1.04-1.06$ & {$\left[\mathrm{~g} / \mathrm{cm}^{2}\right]$} \\
\hline Tensile strength & $32-45$ & {$\left[\mathrm{~N} / \mathrm{mm}^{2}\right]$} \\
\hline Thermal conductivity & 0.18 & {$[\mathrm{~W} / \mathrm{mK}]$} \\
\hline Thermal diffusivity & 1.4 & {$\left[10^{-3} \mathrm{~cm}^{2} / \mathrm{s}\right]$} \\
\hline Specific heat & 1.3 & {$[\mathrm{~kJ} / \mathrm{kg} \mathrm{K}]$} \\
\hline hardness & $85-97$ & \\
\hline Heat deflection temperature & $95-110$ & {$\left[{ }^{\circ} \mathrm{C}\right]$} \\
\hline Coefficient of linear expansion & $60-110$ & {$\left[\mathrm{~K}^{-1} 10^{6}\right]$} \\
\hline Surface tension & $<40$ & {$[\mathrm{dyn} / \mathrm{cm}]$} \\
\hline Viscosity & $>1$ & {$[\mathrm{poise}]$} \\
\hline
\end{tabular}




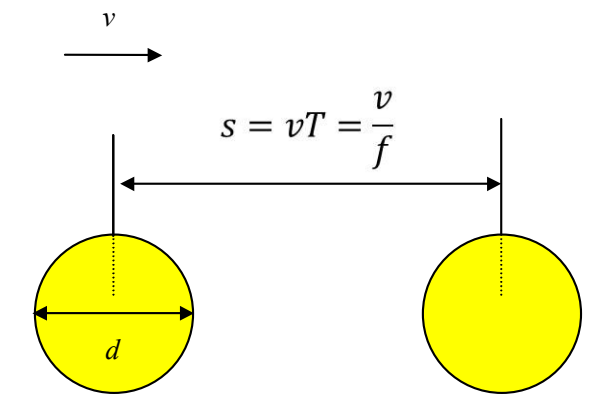

Fig. 2 Distance between two pulses

Table 2 Experimental parameters

\begin{tabular}{|c|c|c|}
\hline $\mathrm{v}[\mathrm{mm} / \mathrm{s}]$ & $\mathrm{s}[\mu \mathrm{m}]$ & Overlap \\
\hline 25 & 0.625 & 23 \\
\hline 50 & 1.25 & 11 \\
\hline 100 & 2.5 & 5 \\
\hline 200 & 5 & 2 \\
\hline 300 & 7.5 & 1 \\
\hline
\end{tabular}

Table 3 The number of scanning passes

\begin{tabular}{|l|l|l|l|l|l|l|l|l|l|l|}
\hline $\mathrm{N}$ & 1 & 2 & 4 & 6 & 8 & 10 & 20 & 30 & 40 & 50 \\
\hline
\end{tabular}

two pulses (s) is expressed as

$$
s=v T=\frac{v}{f}
$$

where $T\left(=\frac{1}{f}\right)$ is a period. Distance between two pulses is depicted in Fig. 2. Scanning speeds, distance between two pulses, and the number of overlap within the spot size are shown in Table 2. Since $\mathrm{v}$ is fast enough, the number of overlap is more than 1 for all scanning speed used for the experiments. The number of scanning passes $(\mathrm{N})$ used in this study is shown in Table 3. Top surface and cross-section of the processed ABS are investigated by microscope. The workpiece is sectioned by a wheel dicing.

\section{Results and Discussions}

Processed workpieces are shown in Fig. 3, When a laser ablation is conducted, surface color is changed from gray or yellow to brown. This may be due to thermal damage to the surrounding area. If more laser energy is applied, surface color is changed from brown to black. In addition, shiny surface along the scanning direction is observed. The color variation shows that laser ablation of the ABS is becoming a photo-thermal dominant process as laser energy increases ${ }^{25}$.

Ablation characteristics such as ablation depth $(D)$ and width $(W)$ are investigated. Depth is plotted in terms of the number of scanning passes as shown in Fig. 4. As the number of scanning passes increases, ablation depth increases logarithmically. Logarithmic trends are also plotted in Fig. 4 and the trend is expressed as follows.

$$
D=a \log (N)+b
$$

where $D$ is depth, and $N$ is the number of scanning passes. In relatively high scanning speed such as $100 \sim 300 \mathrm{~mm} / \mathrm{s}$, depth seems saturated. In relatively low scanning speed such as $25 \sim 50 \mathrm{~mm} / \mathrm{s}$, depth is logarithmically increases. It may saturate too if the number of scanning passes increases under the relatively low scanning speed. This saturation may be due to the plasma formation during laser machining process ${ }^{25)}$. Depth dif-

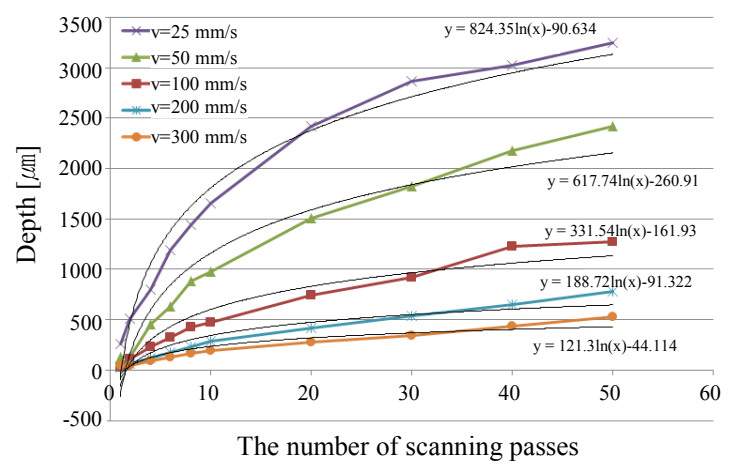

Fig. 4 Depth vs the number of scanning passes

$300 \mathrm{~mm} / \mathrm{s}$

$200 \mathrm{~mm} / \mathrm{s}$

$100 \mathrm{~mm} / \mathrm{s}$

$50 \mathrm{~mm} / \mathrm{s}$

$25 \mathrm{~mm} / \mathrm{s}$
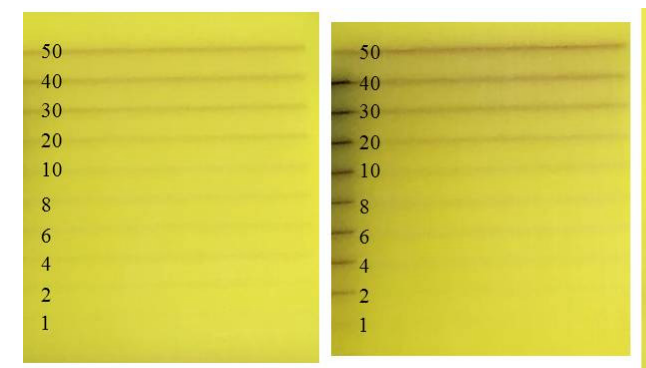

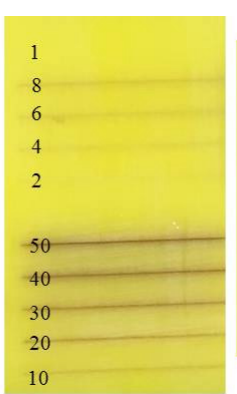

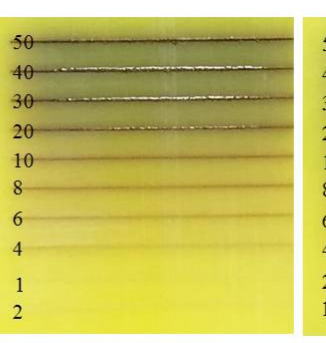

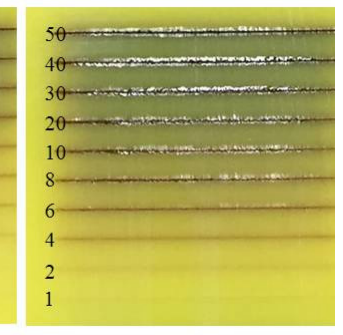

Fig. 3 Top surface of the ablated workpiece at $v=25,50,100,200,300 \mathrm{~mm} / \mathrm{s}$. Numbers in the figure indicate the number of scanning passes 
ferences among the laser scanning speed cases become significant as the number of scanning passes increases. Depth differences between the scanning speed of 25 and $300 \mathrm{~mm} / \mathrm{s}$ when the number of scanning passes is 10 and 50 are 2267.4 and $5062.8 \mathrm{~mm}$. respectively.

According to Eq. (2), coefficients a and b are obtained and tabulated in Table 4 as well as correlation coefficients $\left(R^{2}\right)$ are shown in Fig. 5. As the scanning speed increases, the coefficient a decreases exponentially. On the other hand, the coefficient $\mathrm{b}$ increases almost linearly. According to the correlation coefficients $\left(R^{2}\right)$, it shows very high correlation.

The coefficients a and b also can be curve-fitted as shown in Fig. 5 and can be expressed as follows

$$
\begin{aligned}
& a=1367.6 e^{-0.007 v} \quad\left(R^{2}=0.9344\right) \\
& b=1.2909 v+369.69 \quad\left(R^{2}=0.8027\right)
\end{aligned}
$$

The coefficientsa a and b from the Eq. (2) can be substituted by Eq. (3) and Eq. (4) so that Eq. (2) can be expressed as follows.

$$
D=1367.6 e^{-0.007 v} \log (N)+1.2909 v+369.69
$$

Therefore, Eq. (5) suggests an ablation depth prediction model as a function of scanning speed and the number of scanning passes. 3D plot of the ablation depth prediction model is shown in Fig. 6. It is noted that the suggested ablation depth prediction model may be used to find physically meaningful values as if an incubation model is used to find an ablation threshold ${ }^{26)}$.

Table 4 Curve fitting coefficients of depth

\begin{tabular}{|c|c|c|}
\hline $\mathrm{v}[\mathrm{mm} / \mathrm{s}]$ & $\mathrm{a}$ & $\mathrm{b}$ \\
\hline 25 & 824.35 & -90.63 \\
\hline 50 & 618.31 & -268.19 \\
\hline 100 & 331.54 & -161.93 \\
\hline 200 & 188.72 & -91.32 \\
\hline 300 & 121.30 & -44.11 \\
\hline
\end{tabular}

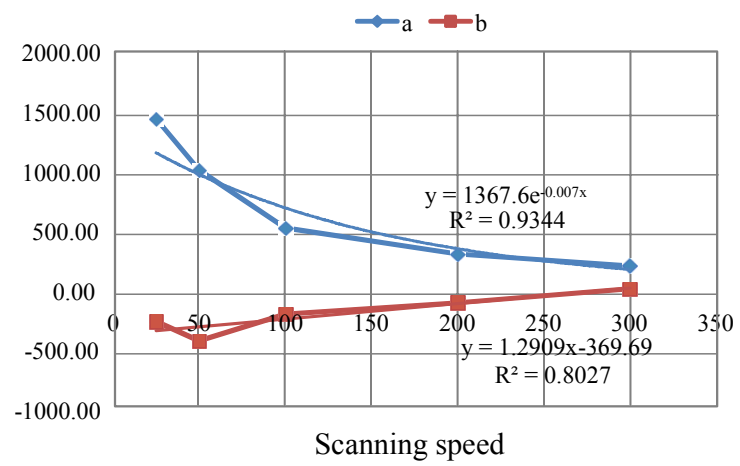

Fig. 5 Curve-fitting coefficient vs. scanning speed

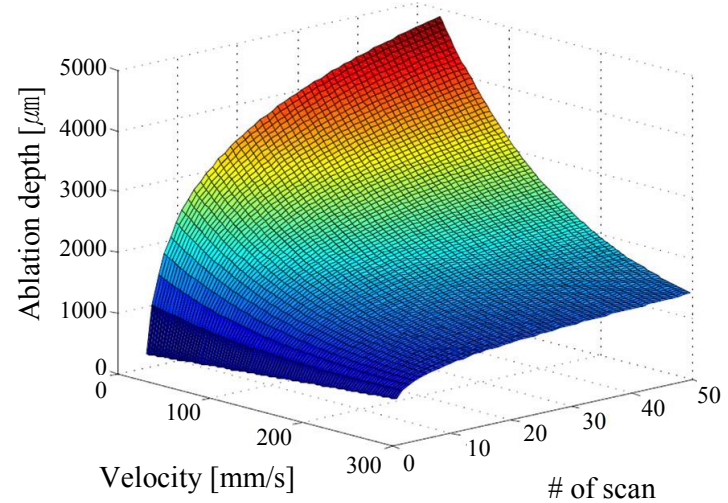

Fig. 6 Ablation depth prediction model as a function of velocity and the number of scanning passes

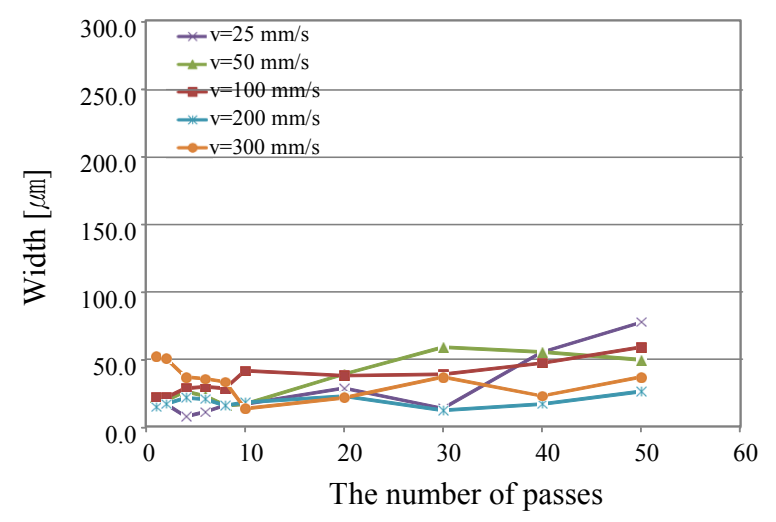

Fig. 7 Width vs the number of scanning passes

The ablation width is shown in Fig. 7 in terms of the number of scanning passes. Even though the ablation width is hard to measure consistently, it is obvious that no significant variation in the ablation width is found compared to the depth under the same scanning speed. The ablation depth is quite deep in comparison of the ablation width, thus, the ablation has a high aspect ratio $(\sim 327.2)$. Since the ABS is a semi-transparent, laser energy can be delivered deep inside the ABS. Furthermore, the absorption coefficient may be increased when surface color is changed from yellow to brown to black. Moreover, as the hole is getting deeper, the number of reflection inside the hole is increased so that the multiple reflection dominantly affects the formation of a deep penetration hole. Thus, the high aspect ratio can be obtained.

To compare all parameters used in this study, the scanning speed and the number of scanning passes are expressed by a specific parameter, namely an accumulated line energy. The accumulated line energy is calculated by following expression

$$
E_{\text {Line }}=\frac{P_{\text {peak }}}{A_{v}} N
$$




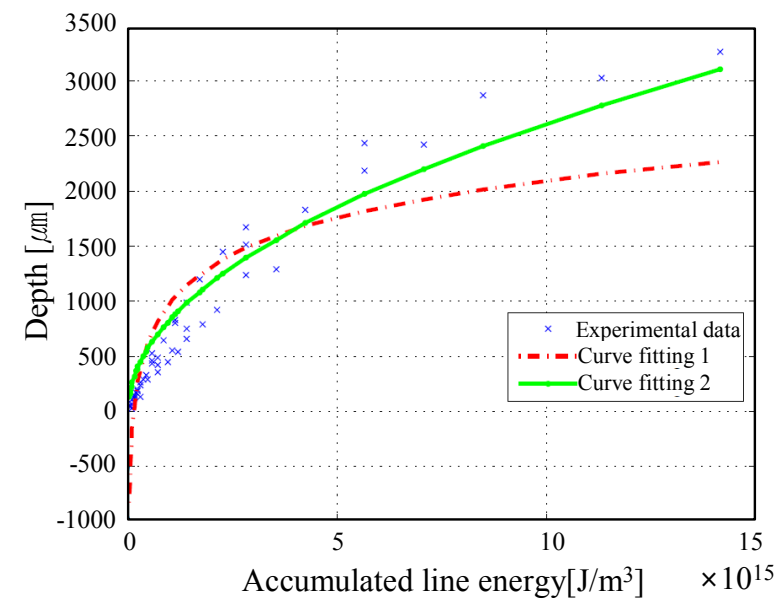

Fig. 8 Depth vs Accumulated line energy

where $\mathrm{A}$ is the area of laser spot size, $\mathrm{v}$ is the scanning speed, and $N$ is the number of scanning passes. Fig. 8 shows depth in terms of the accumulated line energy. Depth increases logarithmically. The curve fitted by a logarithmic function is expressed by

$$
D=540.5342 \log \left(E_{\text {Line }}\right)-12840\left(R^{2}=0.7695\right)
$$

The graph obtained from the logarithmic curve fitting is shown in Fig. 8 and the correlation coefficient $\left(R^{2}\right)$ is 0.7695. To have a better fitting, the curve fitting using a square root function can be used and the result is shown in Eq. (8)

$$
D=1.7375 \times 10^{-6} \sqrt{(15)^{2} E_{\text {Line }}} \quad\left(R^{2}=0.9842\right)
$$

This curve fitting graph is shown in Fig. 8 and the correlation coefficient is 0.9842 . Both graph and the correlation coefficient show that this curve fitting is better than the curve fitting using logarithmic function.

Cross-sections of the ablated workpieces are observed. Cross-sections of the ablated workpiece at $\mathrm{v}=25 \mathrm{~mm} / \mathrm{s}$ are shown in Fig. 9. The width of ablation zone is relatively wide. Especially, at the middle of the ablation depth, the ablation zone is wider than top and bottom of the ablation depth. It may be due to multiple reflections as the ablation zone is deeper. When the number of scanning passes is more than 20, shiny black color can be found. According to the color change, we can estimate absorbed laser energy indirectly

Cross-sections of the ablated workpiece at $\mathrm{v}=100 \mathrm{~mm} / \mathrm{s}$ are shown in Fig. 10. While almost similar phenomena are observed to the scanning speed of $300 \mathrm{~mm} / \mathrm{s}$, debris is more clearly found. If the number of scanning passes is 20 and more, brown color is found at the interface be-
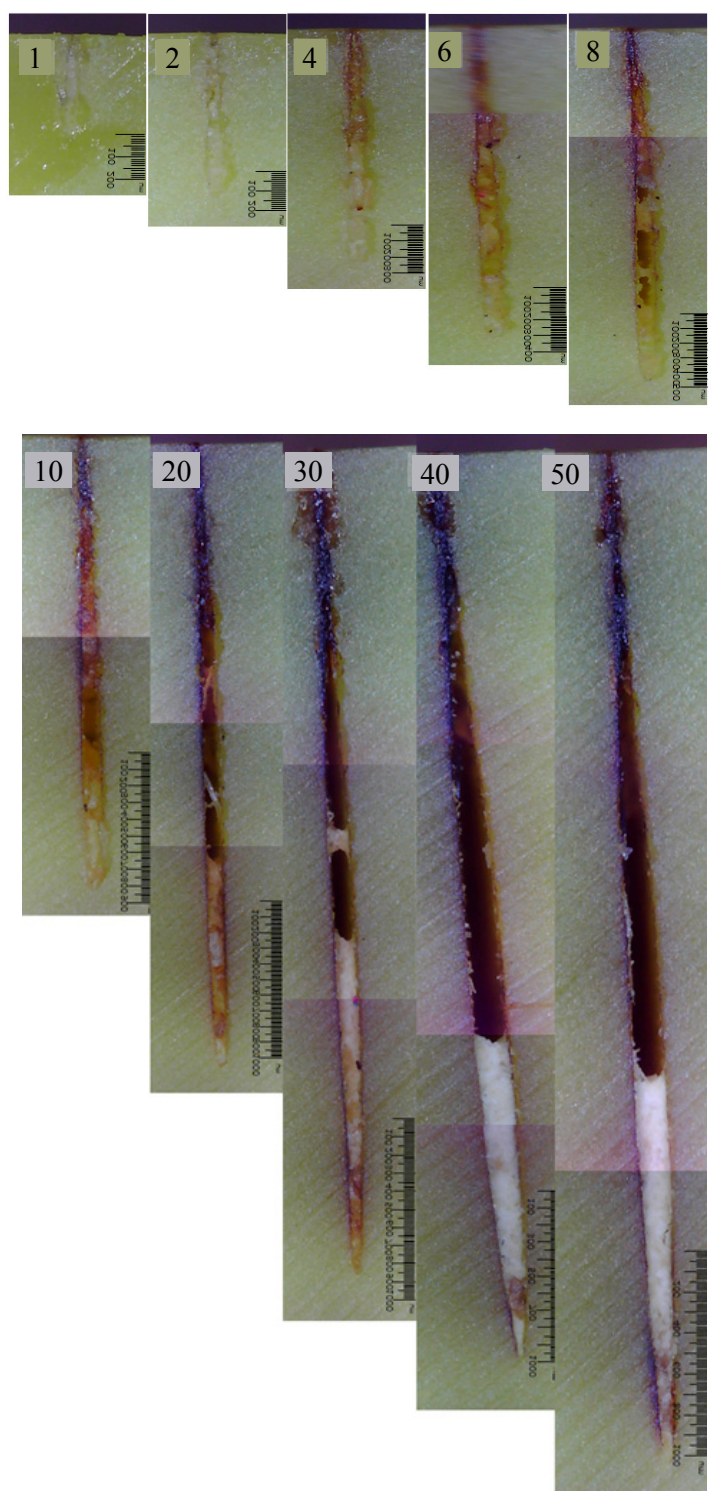

Fig. 9 Cross-section of the ablated workpiece at v=25 mm/s (The number of scanning passes are shown in a box)

tween the ablation zone and base ABS. In this scanning speed, the debris is partially ablated. As the number of scanning passes increases more than 20, the brown color became vivid and even black color is observed.

Cross-sections of the ablated workpiece at $\mathrm{v}=300 \mathrm{~mm} / \mathrm{s}$ are shown in Fig. 11. Under $\mathrm{v}=300 \mathrm{~mm} / \mathrm{s}$, overlap between the laser beam is 1 , which is the sparsest overlap. Due to the sparsest overlap, the case of $v=300 \mathrm{~mm} / \mathrm{s}$ have the least laser intensity among the other cases. When the scanning speed is $300 \mathrm{~mm} / \mathrm{s}$ and the number of scanning passes is less than or equal to 2, no clear ablation zone is observed. Just a notch-like shape along the ablation depth is observed. This indicates that the laser energy is interacted and absorbed by the material. If the number of scanning passes is more than or equal 


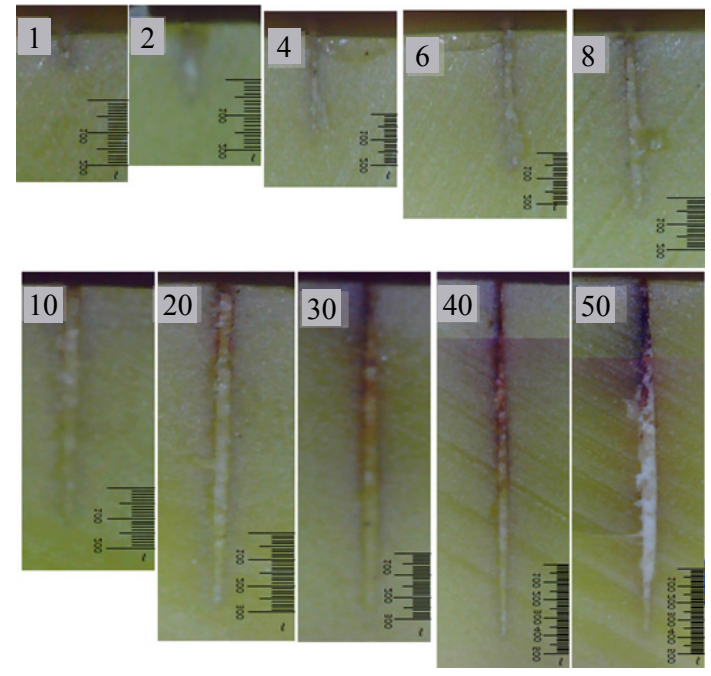

Fig. 10 Cross-section of the ablated workpiece at $v=100$ $\mathrm{mm} / \mathrm{s}$ (The number of scanning passes are shown in a box)

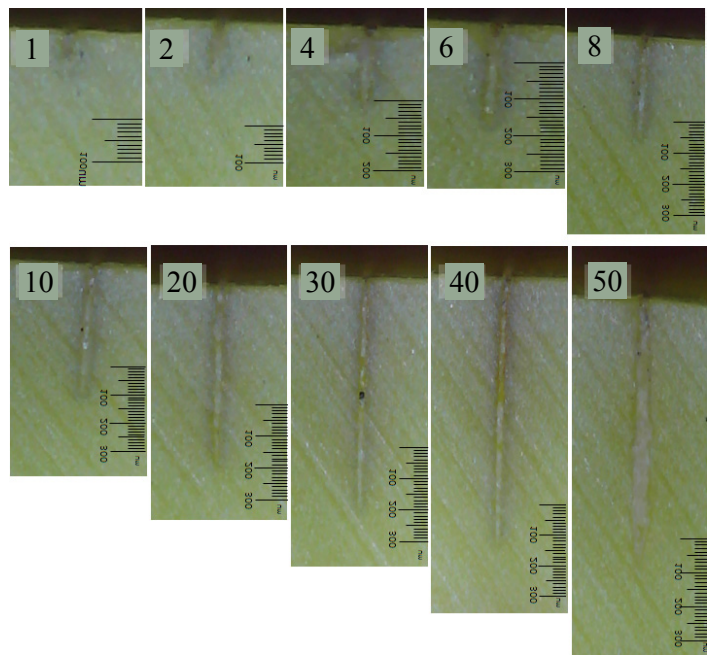

Fig. 11 Cross-section of the ablated workpiece at $v=300$ $\mathrm{mm} / \mathrm{s}$ (The number of scanning passes are shown in a box)

to 4 , we can observe the ablation zone and color change. Gray color is observed around an ablation zone boundary. As the number of scanning passes increases, the ablation zone is fully filled with a redeposited material, or debris. The debris color is white. The author suspect that the debris is deposited during a wheel dicing process, used to observe the cross-section. These phenomena observed from the scanning speed of $300 \mathrm{~mm} / \mathrm{s}$ are also observed in the scanning speed of $200 \mathrm{~mm} / \mathrm{s}$.

\section{Conclusion}

Due to the low degree of precision, long post-processing time, and poor surface roughness under the 3D printing technologies, a laser machining technology to fab- ricate helical structure can be a promising option. This research examines interaction characteristics between a laser and Acrylonitrile butadiene styrene (ABS) used for 3D printing. Laser machining characteristics on this material are investigated with a variation of laser parameters such as a scanning speed and the number of scanning passes. An ablation depth prediction model, or $D=1367.6 e^{-0.007 v} \log (N)+1.2909 v+369.69$, is suggested as a function of a scanning speed and the number of scanning passes. Laser machining on ABS shows a high aspect ratio $(\sim 327.2)$. Furthermore, color changes around the ablation zone can be utilized for the indication to estimate the absorbed laser energy indirectly.

ORCID: Dongkyoung Lee: http://orcid.org/0000-0001-9404-2321

\section{References}

1. B.J. Nelson, I.K. Kaliakatsos, J.J. Abbott, Microrobots for Minimally Invasive Medicine, Annual Review of Biomedical Engineering, 12(1) (2010), 55-85 https://doi.org/10.1146/annurev-bioeng-010510-103409

2. M. Sitti, H. Ceylan, W. Hu, J. Giltinan, M. Turan, S. Yim, E. Diller, Biomedical Applications of Untethered Mobile Milli/Microrobots, Proceedings of the IEEE, 103(2) (2015), 205-224 https://doi.org/10.1109/JPROC.2014.2385105

3. S. Kim, C. Laschi, B. Trimmer, Soft robotics: a bioinspired evolution in robotics, Trends in Biotechnology, 31(5) (2013), 287-294

https://doi.org/10.1016/j.tibtech.2013.03.002

4. S. Jeon, G. Jang, H. Choi, S. Park, Magnetic Navigation System With Gradient and Uniform Saddle Coils for the Wireless Manipulation of Micro-Robots in Human Blood Vessels, IEEE Transactions on Magnetics, 46(6) (2010), 1943-1946 https://doi.org/10.1109/TMAG.2010.2040144

5. K.E. Peyer, S. Tottori, F. Qiu, L. Zhang, B.J. Nelson, Magnetic Helical Micromachines, Chemistry - A European Journal, 19(1) (2013), 28-38

6. K.E. Peyer, L. Zhang, B.J. Nelson, Bio-inspired magnetic swimming microrobots for biomedical applications, Nanoscale, 5(4) (2013), 1259-1272

https://doi.org/10.1002/chem.201203364

7. S.M. Jeon, G.H. Jang, W.S. Lee, Drug-Enhanced Unclogging Motions of a Double Helical Magnetic Micromachine for Occlusive Vascular Diseases, IEEE Transactions on Magnetics, 50(11), (2014) 1-4 https://doi.org/10.1109/TMAG.2014.2320580

8. D. Lee, Experimental Investigation of Laser Spot Welding of $\mathrm{Ni}$ and Au-Sn-Ni Alloy, Journal of Welding and Joining, 35(2) (2017), 1-5 https://doi.org/10.5781/JWJ.2017.35.2.1

9. W. Lee, T. Park, I. Park, Study on a Residual Stress Reduce in Laser Welding Process using Ti6Al4V, Journal of Welding and Joining, 34(6) (2016), 35-41 https://doi.org/10.5781/JWJ.2016.34.6.35 
10. D. Lee, Modeling of High Speed Remote Laser Cutting for Lithium-ion Batteries, Scholar's Press, (2016)

11. D. Lee, J. Mazumder, Effects of laser beam spatial distribution on laser-material interaction, Journal of Laser Applications, 28(3) (2016), 032003 https://doi.org/10.2351/1.4947096

12. D. Lee, R. Patwa, H. Herfurth, J. Mazumder, Parameter optimization for high speed remote laser cutting of electrodes for lithium-ion batteries, Journal of Laser Applications, 28(2) (2016), 022006 https://doi.org/10.2351/1.4942044

13. D. Lee, R. Patwa, H. Herfurth, J. Mazumder, Three dimensional simulation of high speed remote laser cutting of cathode for lithium-ion batteries, Journal of Laser Applications, 28(3) (2016), 032010 https://doi.org/10.2351/1.4950908

14. W.M. Steen, J. Mazumder, SpringerLink (Online service), Laser Material Processing, Springer-Verlag London, London, (2010) http://www.springer.com/kr/book/9781849960618

15. D. Lee, J. Cho, C.H. Kim, S.H. Lee, Application of laser spot cutting on spring contact probe for semiconductor package inspection, Optics \& Laser Technology, 97 (2017), 90-96 https://doi.org/10.1016/j.optlastec.2017.06.005

16. D. Lee, J. Mazumder, Effects of momentum transfer on sizing of current collectors for lithium-ion batteries during laser cutting, Optics \& Laser Technology in press, (2017) https://doi.org/10.1016/j.optlastec.2017.09.016

17. D. Lee, S. Ahn, Investigation of Laser Cutting Width of LiCoO2 Coated Aluminum for Lithium-Ion Batteries, Applied Sciences, 7(9) (2017) https://doi.org/10.3390/app7090914

18. D. Lee, J. Mazumder, Effects of momentum transfer on sizing of current collectors for lithium-ion batteries during laser cutting, Optics \& Laser Technology 99(Supplement C), (2018), 315-325

https://doi.org/10.1016/j.optlastec.2017.09.016
19. B. Neuenschwander, B. Jaeggi, M. Zimmermannn, V. Markovic, B. Resan, K. Weingarten, R. de Loor, L. Penning, Laser surface structuring with $100 \mathrm{~W}$ of average power and sub-ps pulses, Journal of Laser Applications, 28(2) (2016), 022506 https://doi.org/10.2351/1.4944104

20. T. Häfner, J. Heberle, M. Dobler, M. Schmidt, Influences on incubation in ps laser micromachining of steel alloys, Journal of Laser Applications, 28(2) (2016), 022605 https://doi.org/10.2351/1.4944445

21. O. Suttmann, J. Duesing, L. Overmeyer, Phenomenological model for prediction of complex ablation geometries in metal films using ultrashort laser pulses, Journal of Laser Applications, 28(2) (2016), 022208 https://doi.org/10.2351/1.4944507

22. C. Daniel, S. Ostendorf, S. Hallmann, C. Emmelmann, Picosecond laser processing of polycrystalline cubic boron nitride - A method to examine the ablation behavior of a high cubic boron nitride content grade material, Journal of Laser Applications, 28(1) (2016), 012001 https://doi.org/10.2351/1.4931930

23. M. Ilie, E. Cicala, D. Grevey, S. Mattei, V. Stoica, Diode laser welding of ABS: Experiments and process modeling, Optics \& Laser Technology, 41(5) (2009), 608-614 https://doi.org/10.1016/j.optlastec.2008.10.005

24. B. Acherjee, A.S. Kuar, S. Mitra, D. Misra, S. Acharyya, Experimental investigation on laser transmission welding of PMMA to ABS via response surface modeling, Optics \& Laser Technology, 44(5) (2012), 1372-1383 https://doi.org/10.1016/j.optlastec.2011.12.029

25. T. Lippert, Laser Application of Polymers, in, K.T. Lippert (Ed.), Polymers and Light, Springer Berlin Heidelberg, Berlin, Heidelberg, (2004), 51-246 https://doi.org/10.1007/b12682

26. S. Nolte, C. Momma, H. Jacobs, A. T,nnermann, B.N. Chichkov, B. Wellegehausen, H. Welling, Ablation of metals by ultrashort laser pulses, J. Opt. Soc. Am. B, 14(10) (1997), 2716-2722

https://doi.org/10.1364/JOSAB.14.002716 